This is the accepted version of the article:

Navarro-Urrios D., Gomis-Bresco J., Alzina F., Capuj N.E., García P.D., Colombano M.F., Chavez-Angel E., Sotomayor-Torres C.M.. Self-sustained coherent phonon generation in optomechanical cavities. J ournal of Optics (United Kingdom), (2016). 18. 094006: - . 10.1088/2040-8978/18/9/094006.

Available at:

https://dx.doi.org/10.1088/2040-8978/18/9/094006 


\section{Self-sustained coherent phonon generation in optomechanical cavities}

D. Navarro-Urrios ${ }^{1}$, J. Gomis-Bresco ${ }^{1,2}$, F. Alzina ${ }^{1}$, N. E. Capuj ${ }^{3}$, P. D. García ${ }^{1}$, M. F. Colombano ${ }^{1}$, E. Chavez-Angel ${ }^{1,4}$, C. M. Sotomayor-Torres ${ }^{1,5}$

${ }^{1}$ Catalan Institute of Nanoscience and Nanotechnology (ICN2), CSIC and The Barcelona Institute of Science and Technology, Campus UAB, Bellaterra, 08193 Barcelona, Spain

2 ICFO-Institut de Ciencies Fotoniques and Universitat Politécnica de Catalunya, Mediterranean Technology Park, 08860 Castelldefels (Barcelona), Spain

${ }^{3}$ Depto. Física. Universidad de la Laguna. 38206. Spain

${ }^{4}$ Institute of Physics, University of Mainz, Staudinger Weg 7, 55128 Mainz, Germany

${ }^{5}$ Catalan Institution for Research and Advanced Studies (ICREA), 08010 Barcelona, Spain

\section{Abstract}

Optical forces can set tiny objects in states of mechanical self-sustained oscillation, spontaneously generating periodic signals by extracting power from steady sources. Miniaturized self-sustained coherent phonon sources are interesting for applications such as mass-force sensing, intra-chip metrology and intra-chip time-keeping among others. In this paper, we review several mechanisms and techniques that can drive a mechanical mode into the lasing regime by exploiting the radiation pressure force in optomechanical cavities, namely stimulated emission, dynamical back-action, forward stimulated Brillouin scattering and selfpulsing.

\section{Introduction}

Phonon or mechanical lasing can be defined as a regime in which the mechanical oscillations are self-sustained, monochromatic, coherent and of high-amplitude [1]. Just as for conventional lasers, phonon lasers display a power threshold above which the passive mechanical losses are compensated and the overall damping rate becomes negative. Oscillations thus grow exponentially until a steady-state regime is reached when non-linear losses compensate gain. Above the threshold, the mechanical signal displays linewidth narrowing that could be described by a Schawlow-Townes-type limit [2]. Contrary to conventional lasers, mechanical gain does not have to originate necessarily from a stimulated emission mechanism. In this work we present several strategies for achieving this regime in optomechanical (OM) cavities using the radiation pressure as the driving force of the motion. 
In the phonon lasing regime, the micro/nano-mechanical resonator can be used as an integrated photonic clock or a reference oscillator [3]. The latter could eventually become part of a network of oscillators to spread a common clock signal by exploiting synchronization among several oscillators [4]. Indeed, there are already experimental evidences of synchronization of two OM phonon lasers in the classical regime, using optically coupled microdisks [5], as well as in an optical racetrack cavity integrated with two mechanical oscillators [6]. More recently, a larger array of up to seven optically coupled microdisks were used to demonstrate phase noise reduction due to synchronization [7]. However, a note of caution has been sounded regarding the previous systems [8] as they rely on strong optical coupling, thus being unclear the difference between synchronization of individual oscillators and the excitation of a single coupled mode. Further applications of phonon lasers can be also found in mass sensing, since at room temperatures (RT) and/or in fluidic environments, micro/nano-mechanical resonators suffer from very low quality factors and the devices must be driven to reduce frequency noise.

High-amplitude coherent phonon signals have been generated by introducing an external positive feedback, in a manner akin to that reported in Ref. [9] for electromechanical systems or directly phase-modulating the input laser light resonantly with a mechanical mode [10]. However, in the latter case, the resonator is treated as a passive one, characterized by a damped response to impulsive stimuli. Here we focus on active resonators, i.e., those in which the self-sustained regime is spontaneously activated by extracting energy from continuous wave laser sources.

The manuscript is divided in five sections: the first four review different pumping schemes that have been used to reach the phonon lasing regime while the last section addresses passive mechanical loss channels of different OM cavity setups. Regarding the first sections, we start with a lasing mechanism that rely on population inversion among different optical energy levels. The second section deals with, probably, the most common pumping mechanism for phonon lasing, namely dynamical back-action. Stimulated forward Brillouin scattering in circular microresonators is discussed in the third section. The fourth section is focused on selfpulsing limit cycles, which are spontaneous dynamical processes that modulate the intracavity radiation pressure force. In addition to a literature review, we further analyze two of the mechanisms discussed in the manuscript, namely dynamical back-action and self-pulsing, in terms of results obtained in the very same OM photonic crystal. 
Within the wide set of OM cavity setups, along the manuscript we pay special attention to circular microresonators and OM photonic crystal cavities. The former were the pioneer setups demonstrating phonon lasing action with the first three mechanisms discussed in this review while the latter have the advantage of being chip-integrated in a way that allow the straightforward connection to phononic waveguides or membranes for out-coupling the phonon lasing signal [10].

\section{Phonon analog of a conventional laser}

In a conventional laser, stimulated emission occurs from upper to lower energy states of atoms and ions. The first mechanical analogue to the optical laser was demonstrated on a single magnesium ion, held in a linear radio-frequency trap and irradiated by two laser beams tuned below (red detuned) and above (blue detuned) an atomic resonance, respectively. For appropriate settings of laser detunings and intensities, regenerative oscillations with a stable amplitude start from noise [11].

Inspired by the works done on trapped ions, the phonon analog of a two-level laser system using OM cavities was demonstrated by Grudinin et al. [12] based on a compound microcavity system coupled to a radio-frequency mechanical mode. The main idea lies in optically coupling a pair of nominally equivalent OM cavities in a way that symmetric and antisymmetric optical supermodes are created out of the degenerate modes of the isolated cavities. Due to unavoidable accuracy limitations in the fabrication stage, identical cavities are difficult to realize and the optical resonances of the individual cavities become slightly detuned from each other. This is generally solved by heating one of them with an out-of-plane laser, thus increasing its refractive index thanks to thermo-optic (TO) effects. With this method it is possible to control the coupling strength between both cavities, enabling the fine tuning of the frequency difference of the two optical supermodes until it is resonant with a mechanical eigenfrequency $\left(\Omega_{\mathrm{m}}\right)$ of either one of the cavities. Population inversion is achieved by resonantly exciting the upper optical level with another laser, from where the system relaxes down to the lower optical level by emitting a resonant phonon (Fig. 1). Population inversion produces mechanical gain, causing phonon laser action above a pump power threshold, which in the case of Ref. [12] was a few $\mu \mathrm{W}$.

This kind of systems features a continuously tunable gain spectrum to amplify selectively mechanical modes from radio frequency to microwave rates. In a Brillouin scattering picture, the system accesses a regime in which the phonon plays the role of the Stokes wave. For this 
reason, it should also be possible to switch in a controlled way between phonon and photon laser regimes.

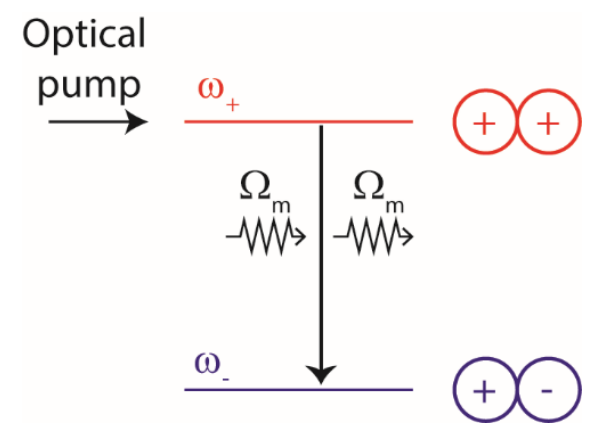

Fig. 1. Two-level phonon laser energy level diagram including schematics of the photonic symmetric $(w+)$ and antisymmetric ( $(w-)$ modes. The phonon frequency is denoted by $\Omega_{m}$.

As stated in the introduction, a similar scheme of optically coupled cavities has been recently realized to activate the mechanical lasing regime in up to seven coupled microdisks that synchronize their oscillations [5,7]. However, in those works, the OM cavities are driven by blue-detuned continuous wave lasers, i.e., the energy of the laser photons is higher than that of the cavity resonance. Therefore, the self-sustained mechanical oscillations are generated via the dynamical back-action between optical and mechanical modes, which is discussed in the following section.

It is worth to mention here that a direct analog to an optical laser based on a three-level system has been demonstrated recently on an electromechanical system [13]. The device relies on a purely mechanical architecture, where three phononic states are involved in addition to the phonon thermal bath, which act as the first level. The energy difference between the two higher energy modes is resonant with a lower energy mode, which is the one displaying the phonon lasing features.

\section{Dynamical back-action}

One of the basic consequences of the OM interaction in OM cavities is dynamical back-action, which is a result of the retarded response of the radiation pressure force to mechanical deformation. The mechanism can also be understood in terms of a scattering process, where Stokes and anti-Stokes sidebands appear as a consequence of the OM interaction. If the laser is detuned with respect to the cavity resonance by an amount equal to $\Delta$ (in frequency units), one can obtain an asymmetric spectrum to allow a net energy transfer from light to the mechanical mode or vice versa. In order to achieve effective mechanical amplification or cooling, the cavity needs to be within the sideband-resolved regime $\left(\Omega_{m}>\kappa, \kappa\right.$ being the overall 
radiative damping rate) and the laser has to be blue-detuned ( $\Delta>0$, heating) or red-detuned $\left(\Delta<0\right.$, cooling), respectively. The general expression for the OM damping rate $\left(\Gamma_{O M}\right)$ under the linear approximation of a strong coherent laser driving tone can be derived from the Langevin equations associated to the OM Hamiltonian [1]:

$\Gamma_{O M}\left(\Omega_{m}\right)=g_{o, O M}^{2} n_{o}\left[\frac{\kappa}{\left(\Delta+\Omega_{m}\right)^{2}+\kappa^{2} / 4}-\frac{\kappa}{\left(\Delta-\Omega_{m}\right)^{2}+\kappa^{2} / 4}\right] \quad$,

, where $g_{o, O M}$ is the single-particle OM coupling rate, and $n_{0}$ is the number of intracavity photons. In Fig. 2 we have plotted $\Gamma_{O M}$ according to Eq. 1 as a function of the normalized detuning $\left(\Delta / \Omega_{m}\right)$ and the normalized mechanical frequency $\left(\Omega_{m} / \kappa\right)$. The overall mechanical damping rate $\left(\Gamma_{m}\right)$ is given by the accumulation of the intrinsic damping rate $\left(\Gamma_{m, i}\right)$ and $\Gamma_{\mathrm{OM}}$, i.e., $\Gamma_{m}=\Gamma_{m, i}+\Gamma_{O M}$. Hence, it is possible to achieve phonon lasing, i.e., compensation of the intrinsic mechanical losses $\left(\Gamma_{m}=0\right)$, only when $\Gamma_{m, i}=-\Gamma_{O M}$. Inspecting Eq. 1 , it is clear that the latter condition is fulfilled more easily at $\Delta=\Omega_{m}$. In particular, for systems well within the sideband-resolved regime $\left(\Omega_{m}>>\kappa\right)$, the contribution of the first term of Eq. 1 can be neglected and phonon lasing is achieved if the field-enhanced cooperativity, defined as $C=n_{o} \frac{4 g_{o, O M}^{2}}{\kappa \Gamma_{m, i}}$, is equal to unity [14]. Phonon lasing by means of dynamical back-action was first reported using microtoroidal cavities [15], demonstrating that in a blue-detuned finite spectral range the linewidth of the mechanical resonance vanished, i.e., a feature of lasing action. Since then, the regime of mechanical lasing has been achieved on a large variety of OM systems such as OM crystals [16], microwave circuits [17], micromirrors [18] or nanostrings [19] among others. 


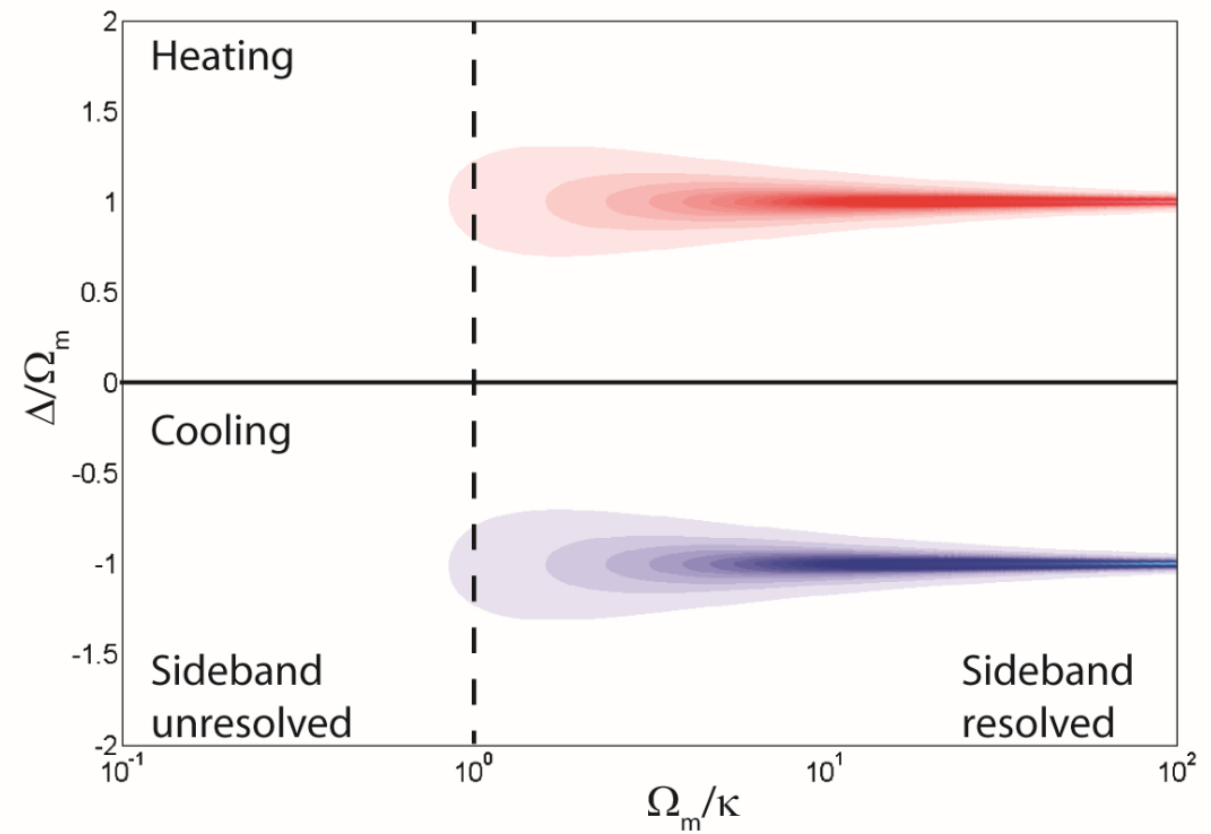

Fig. 2. OM damping rate as a function of normalized detuning and normalized mechanical frequency for a fixed number of intracavity photons and a given OM coupling rate. Red and blue colors denote negative and positive values, respectively).

The dynamical back-action mechanism in a blue-detuned pumping scheme can also be exploited for achieving other functionalities. One example is the amplification of a probe microwave signal in an on-chip microwave cavity parametrically interacting with a micromechanical resonator operating close to the quantum limit [17]. In this proof-ofprinciple device the pump power controls the gain for the input signal via the tailoring of the effective mechanical damping rate and is kept below the mechanical lasing threshold to avoid instabilities of the coupled system. Another example is the "anomalous cooling" of competing mechanical modes in a multimode mechanical resonators [20]. Indeed, just as in a photon laser, a phonon laser can exhibit mode competition. All of the modes within the gain bandwidth initially experience mechanical gain, and those for which the gain exceeds the losses start to oscillate. For sufficiently large oscillations, one of the modes prevail and the gain of the other modes switches sign and becomes damped.

At large oscillation amplitudes, non-linear OM interactions start playing a role. In fact, theoretical predictions of a more complex, multistable attractor diagram [21] have been recently verified experimentally [22], giving rise, for instance, to stable self-sustained oscillations in a red-detuned configuration. 
In order to illustrate the requirements for achieving efficient dynamical back-action effects in ambient conditions of pressure and temperature, we have investigated this mechanism in onedimensional OM crystals fabricated using state-of-the-art silicon (Si) nanofabrication techniques $[23,24]$. We have studied a mechanical mode at $\Omega_{m} / 2 \pi=5.5 \mathrm{GHz}$, which is sidebandresolved $\left(\Omega_{m} / \kappa_{2}\right)$, and exhibits an experimental single-particle OM coupling rate of $\left|g_{o, O M}\right|=1.8 \mathrm{MHz}\left(\left|g_{o, O M}\right| / 2 \pi=0.3 \mathrm{MHz}\right)$ when coupled to the first optical mode supported by the OM cavity. It is worth noting that the latter value is only a factor of 3-4 below the state-ofthe-art in OM crystals [25]. The input laser power was the maximum provided by our tunable laser, i.e., about $1.3 \mathrm{~mW}$.

The mechanical quality factor $\left(Q_{m, i}\right)$ of that mode at RT is $Q_{m, i}=1600$, which corresponds to an intrinsic damping rate $\Gamma_{m, i}=21 \mathrm{MHz}$. On Ref. 24 we reported that, by decreasing $\Delta$, there is an associated decreasing of the damping rate measured to be $\Gamma_{m}=49 \mathrm{KHz}$ at $\Delta / 2 \pi=5.8 \mathrm{GHz}$ (Fig. 3). This represents a reduction of almost three orders of magnitude with respect to the intrinsic value and is consistent with the calculated value using Eq. 1. However, although the mechanical motion associated to this mode has been greatly amplified and its coherence increased, $\Gamma_{m, l}$ was not totally compensated. This is in part because of the spontaneous activation of a self-pulsing (SP) mechanism [33], discussed in the fourth section, which prevented achieving the resonance condition of Eq. 1. If the resonance condition for optimum energy transfer had been achieved, the maximum value of $\Gamma_{O M}$ calculated with Eq. 1 would just be few tens of $\mathrm{KHz}$ above the intrinsic value.

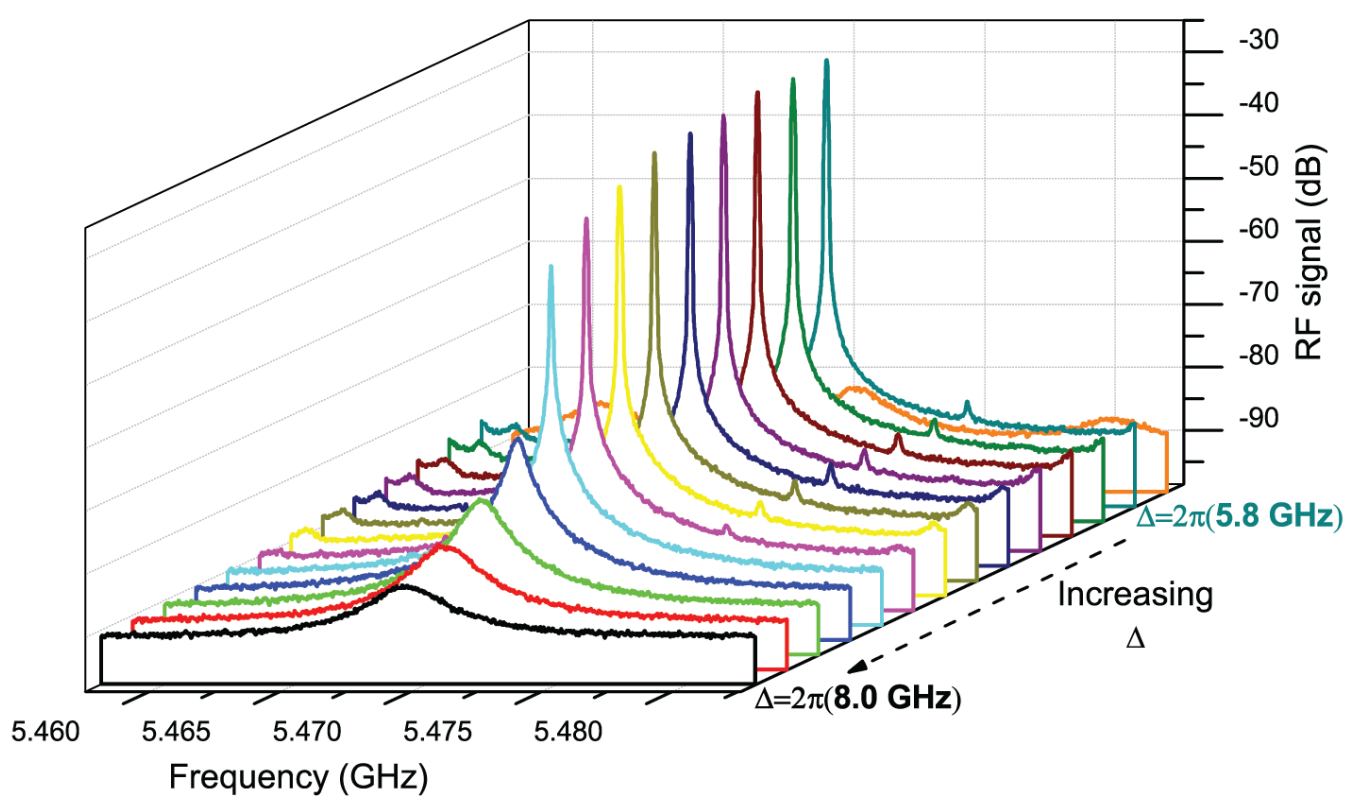


Fig. 3. Mechanical mode at $5.5 \mathrm{GHz}$ optically amplified through a dynamical back-action process. Different curves correspond to different values of laser-cavity detuning $\Delta$.

The previous case of study illustrates that $\Gamma_{m, l}$ can be compensated by means of dynamical back-action mechanism only if the specific optical and mechanical modes of the eventual OM cavity have excellent characteristics in terms of quality factors and OM coupling rates. There are at least two strategies to reduce the power threshold for lasing in OM cavities: (i) fabricating samples with extreme levels of perfection and surface control to lower the intrinsic optical damping rates and (ii) lowering the operational temperature to decrease the intrinsic mechanical damping rate. However, both strategies impact on the degree of applicability of the eventual phonon source.

When the system is within the sideband-resolved regime, only one sideband can lie inside the cavity linewidth. In this regime, only one phonon is exchanged between the optical field and the resonator. On the contrary, if the width of the cavity spectrum is much larger than the mechanical frequency, many sidebands can lie inside the cavity linewidth. In this unresolved sideband regime, more than one phonon can be excited in a blue-detuned configuration (the optimum detuning is a multiple fraction of the mechanical frequency). This feature was exploited in Ref. [34] to experimentally drive a nanomechanical resonator into a regime of oscillation amplitudes equivalent to more than $10^{12}$ phonons. Given that the oscillator has two stable configurations at rest and can be controllably cooled afterwards towards each of the relaxed states, it behaves as a non-volatile optical memory element [34]. However, since the multiphonon scattering process is a quite unlikely event, energy cost of each data operation is still significantly higher than for modern CMOS devices ( $10 \mu \mathrm{J}$ compared with $\sim 1 \mathrm{pJ})$ [35].

We have already shown that producing high-quality optical cavities to exploit radiation pressure back-action is quite challenging. In spite of this, it is still possible to observe OM effects of retarded radiation forces in OM setups where the forces are of photothermal origin. In this scheme, absorption of light can heat the structure and deform it, effectively leading to effects equivalent to those of a force. Optical quality factors are less important here since the thermal relaxation time of the structure replaces the cavity photon lifetime when evaluating the back-action effect. Indeed, self-induced mechanical oscillations have been reported as a consequence of photothermal forces in different OM setups [36-38], which are not necessarily optical cavities.

There are other types of dynamical back-action mechanisms in OM systems that do not require an optical cavity to reach the lasing regime. For instance, in ref. [39], an n-GaAs=i-GaAs bilayer 
microcantilever exhibits mechanical self-oscillations in response to carrier excitation with nearband-gap photon energies, the OM coupling being based on the piezoelectric effect that is generated by photoinduced carriers.

\section{Forward Stimulated Brillouin Scattering}

As discussed in the previous section, dynamical back-action can be described in terms of a sideband-assisted scattering process in which the pump laser is blue shifted from the resonance. A different path for cavity optomechanics to reach the phonon lasing regime is by means of a forward stimulated Brillouin scattering (FSBS). Contrary to dynamical back-action, through FSBS the pump is scattered to only one Stokes sideband since the travelling acoustic resonance acts as a grating continuously moving away from the pump. This was demonstrated for the first time in silica microspheres of few hundreds $\mu \mathrm{m}$ diameter [40]. The stimulated mechanical whispering gallery modes generated by this mechanism are surface acoustic waves of the form typically excited using metal electrodes on piezoelectric substrates. In this case, however, the electrodes are virtual, and are written by light traveling with the mechanical mode. The FSBS process involves two optical modes whose energy differs by the acoustic resonance frequency. Suppression of anti-Stokes scattering is obtained by exploiting the aperiodic spacing between high transverse order optical modes in the resonator, i.e., the scattering mechanism occurs between modes having different radial and/or polar mode number. A similar scheme was used in a contemporary work by Savchenkov et al. [41] using $\mathrm{LiTaO}_{3}$ and $\mathrm{MgF}_{2}$ resonators. In that case the large magnitude of the generated acoustic waves resulted in nonlinear generation of mechanical frequency combs, leading to effects akin to those associated to the Kerr non-linearity in optics.

\section{Self-pulsing mechanisms}

In this section we discuss self-pulsing (SP) mechanisms in optical cavities. These are spontaneous processes triggered within the optical cavity that can modulate the effective refractive index of a localized optical mode and, in turn, the intracavity radiation pressure forces. In optical resonators, when several optical non-linear mechanisms contribute to the effective refractive index of a mode, the long-term solution is, in most of the cases, a stable fixed point. If the involved physical magnitudes (free carrier population, cavity temperature, etc.) are driven away from that point, the system settles down to equilibrium through exponentially damped oscillations. However, for specific values of the parameters governing the non-linear dynamical equations, the stable fixed point may undergo a supercritical Hopf bifurcation, mutating to an unstable fixed-point surrounded by a stable SP limit-cycle. In this 
case, the SP limit-cycle regime is the asymptotic solution towards which the non-linear system settles down.

When a SP limit-cycle is active, the light within the cavity is modulated in a strongly anharmonic way, creating an "optical frequency comb" with multiple frequency peaks spectrally located at integers of the main SP frequency $\left(v_{S P}\right)$. The dynamic behavior of the intracavity photon number is then equivalent to what could be reached by externally modulating the laser input [42]. Thus, this feature can be exploited to pump resonantly a mechanical mode of an OM cavity. Stable SP regimes operating at RT have been reported in optical resonators as a consequence of fast-slow mechanisms of different origins. For instance, Baker et al. [43] reported limit-cycles with periodicity ranging from hundreds of milliseconds to hundreds of seconds as a consequence of the interplay between the thermo-optic effect (fast mechanism) and a thermo-mechanic nonlinearity (slow mechanism) in a Si Nitride microdisk. A similar SP behavior caused solely by the TO nonlinearity in fused silica microspheres was also observed and analyzed in Ref. [44]. In Ref. [45], self-sustained gigahertz electronic oscillations were reported resulting from coupled electron-photon dynamics in ultrahigh-Q Si microdisk resonators with continuous wave pumping.

Another type of SP limit-cycle, which is the one involved along the rest of this section, derives from a stable dynamic competition between Free-Carrier Dispersion (FCD) and thermo-optic (TO) effects [46]. In the case of FCD, the excess of free-carriers leads to a reduction of the material refractive index and therefore a blue-shift of the optical cavity mode [47]. On the other hand, the TO effect results in an increase of the refractive index of the material with increasing temperature [48]. Since the main source of heating is Free-Carrier Absorption (FCA), the dynamics of free-carrier density $(N)$ and the temperature increase $(\Delta T)$ are linked by $n_{0}$. SP limit-cycles of a similar kind have been observed in various photonic structures, such as microdisks and photonic crystals [46,49-52].

The coupling between SP limit-cycles and mechanical degrees of freedom through radiation pressure forces was first tackled in Ref. [33], where phonon lasing was demonstrated in a photonic OM crystal cavity in response to a frequency-tuneable SP limit-cycle. Indeed, as briefly state above, when the SP limit-cycle is active, the radiation pressure optical force $\left(F_{o}\right)$ is modulated in the same way as the $n_{o}$, since they are related in a linear way, i.e., $F_{o}=\hbar$

The mechanical modes of the OM system can be described as damped linear harmonic oscillators driven by $F_{o}$. Finally, the nonlinear resonance position has to include now the effect of the mechanical motion, which is just the OM coupling. 
The SP/OM-cavity non-linear system is thus an indecomposable system coupled through $n_{o}$. Much more complex trajectories than those derived from isolated SP can now arise, opening the way to chaotic trajectories within a basin of attraction in the phase space [53]. The most significant feature in the context of this review is that self-sustained mechanical oscillations are achieved if one of the low harmonics of the SP main peak at $v_{S P}$ is resonant with a mechanical eigenstate $\left(v_{s p}=\Omega_{m} / M\right.$, where $\left.M \in \mathbb{Z}\right)$. In fact, the coherence of the mechanical oscillation is maintained since the mechanical mode lifetime is much longer than $1 / M v_{s p}$.

In the following, we focus on a flexural mode of the very same one-dimensional OM crystal reported in the previous section, whose characteristics are $\Omega_{m}=54 \mathrm{MHz}, g_{0,0 M}=0.3 \mathrm{KHz}$ and $\Gamma_{m, i}=700 \mathrm{KHz}$. The optical mode used in this case had a damping rate of $k=56 \mathrm{GHz}$. In Figure 4, we report the temporal behavior of the inverted transmitted signal for three different laser wavelengths above the threshold for SP. In those, a coherent mechanical oscillation at $\Omega_{m}$ is superimposed on a SP trace at frequencies $v_{\mathrm{SP}}=\Omega_{m} / 2, v_{\mathrm{SP}}=\Omega_{m} / 3$ and $v_{\mathrm{SP}}=\Omega_{m} / 4$ (bottom, medium and top panel respectively), the two systems being locked both in frequency and phase. The sinusoidal-like oscillations correspond to the mechanical coherent oscillation while the strongly asymmetric peaks are mostly associated to the SP, with a smaller contribution from the mechanical oscillation as well. Here it becomes evident that the mechanical oscillator is not only pumped resonantly using the $\mathrm{M}^{\text {th }}$ harmonic of $F_{o}$, but also that the large amplitude of the coherent mechanical motion acts as a feedback that stabilizes and entrains the SP and the mechanical oscillator. Since $\Omega_{\mathrm{m}}$ is much more robust than $v_{\mathrm{sp}}$, the SP mechanism adapts its frequency to the mechanical one. When the resonant condition with the mechanical oscillation is not fulfilled the conventional SP limit-cycle is recovered and the mechanical states are populated only thermally. 


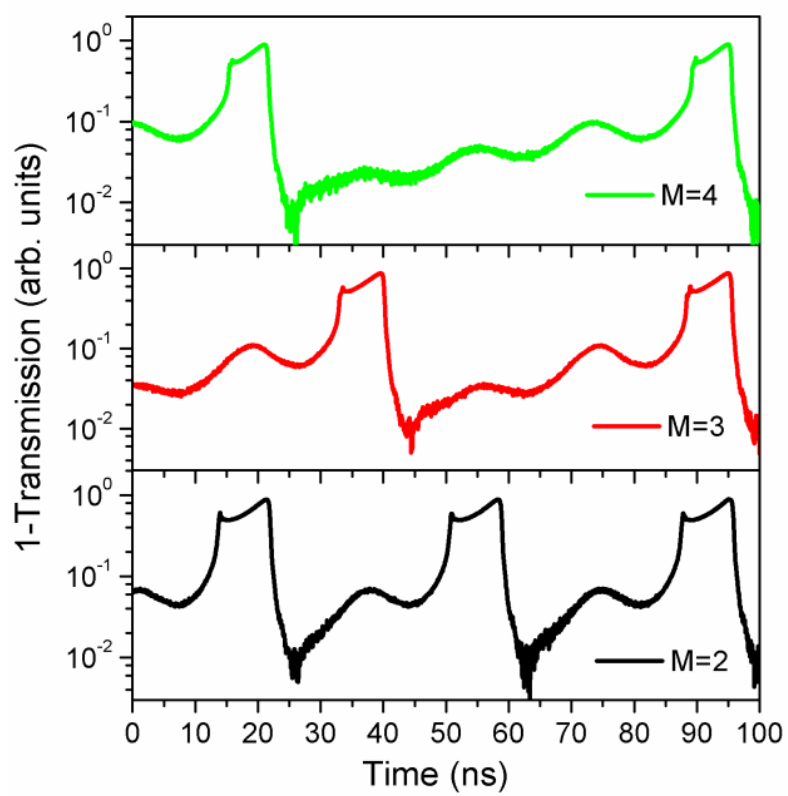

Fig. 4. Dynamic behavior of the inverted transmitted optical signal for three different values of $v_{s p}$. In the three cases, the SP is frequency-entrained with the coherent mechanical oscillation of a flexural mode at $\Omega_{m}=54 \mathrm{MHz}$, which was activated by the anharmonic modulation of the intracavity radiation pressure force. The bottom, medium and top panels correspond to $M=2$, $M=3$ and $M=4$ situations.

The SP/OM-cavity non-linear system described above must not be confused with that behind the observed thermo-mechanical oscillations in optical resonators $[54,55]$. In those works, the mechanical oscillations are of much smaller frequency (up to tens of $\mathrm{KHz}$ ) and are associated to the thermal expansion of the cavities, i.e., contrary to what illustrated in Fig. 4, they are not related to the coherent population of a specific mechanical mode of the structure.

It is instructive to substitute the parameters corresponding to the mechanical mode studied in this section in Eq. 1 to estimate the maximum OM gain at RT that could be obtained by means of dynamical back-action. Since the system is deeply in the unresolved regime, the calculated damping rate is only $\Gamma_{O M}=-2 \times 10^{3} \mathrm{~Hz}$ at $\Delta=\Omega_{m}$. This is more than two orders of magnitude lower than $\Gamma_{l}$, the overall damping rate being thus barely modified with respect to the intrinsic value. On the contrary, we have demonstrated in this section that a SP pumping mechanism achieves phonon lasing at RT despite the modest characteristics of the pair of optical and mechanical modes under study. However, in spite of being more efficient, the current drawback of SP mechanism used in Ref. [33] with respect to dynamical back-action is found on its relatively low operation frequency (tens of $\mathrm{MHz}$ ), which currently limits the frequency of the mechanical modes susceptible to reach the lasing regime to few hundreds of $\mathrm{MHz}$. Further speeding up of 
the SP dynamics could be reached by increasing the heat outflow and/or the free-carrier recombination rates. With the same goal in mind, it would also be possible to exploit faster SP mechanisms, such as that reported by Soltani et al. in Ref. [45].

\section{Mechanical damping mechanisms in OM cavities}

As previously discussed, at the power threshold for phonon lasing the optomechanically induced mechanical damping rate compensates the contribution of intrinsic loss mechanisms, where we include the passive loss channels (material, geometrical and environmental). Low intrinsic rates are obviously leading to low threshold, highly efficient phonon lasers. In this section we will briefly discuss the physical origin of the intrinsic rates in the different OM cavity setups discussed along the manuscript. It is worth noting before starting that gas damping mechanisms are dominant in many OM systems, whose contribution is proportional to the ratio $m_{\text {eff }} \Omega_{m} / p$ ( $m_{\text {eff }}$ is the effective mass of the mechanical oscillator and $p$ is the gas pressure) and depend on the specific geometry. Therefore, most of the literature deals with OM systems embedded within a high vacuum environment.

Concerning circular microresonators, optically levitated nanospheres provide extreme mechanical isolation and ultralow dissipation, which are only limited by gas-molecule collisions in high vacuum, potentially reaching mechanical Q-factors of $10^{12}$ [56]. In clamped circular microresonators, clamping losses are usually the dominating mechanism. The best values in this regard are those reported in silica whispering gallery mode microtoroids suspended by spokes [57]. Mechanical Q-factors of more than $2 \times 10^{4}$ were obtained for modes at $80 \mathrm{MHz}$ at $1 \mathrm{~K}$, which were dominated by two-level fluctuators. The damping rate increased by a factor of 2 rate at room temperature, which was associated to thermo-elastic losses. In any case, for this optimized spoke-supported design, clamping losses were mitigated to the level of being negligible with respect to the other intrinsic mechanisms.

Regarding OM crystals, the mechanical Q-factors obtained at RT are of the order of $10^{3}$, both in a Si [26] and in a Si Nitride platform [27]. The dominant damping mechanism in the GHz range, even at atmospheric pressures, is likely to be scattering with thermal phonons [28], although thermo-elastic damping and clamping effects can also strongly contribute to losses [26]. Gas damping is prevailing only for the extended $\mathrm{MHz}$-tens of $\mathrm{MHz}$ modes at atmospheric pressures. If clamping effects are not dominant, a valid approach to increase $Q_{m, l}$ is to decrease the temperature of the cavity. For low enough cavity temperatures $(<100 \mathrm{~K}$ in ref. [26]), either clamping or surface damping become the dominant loss mechanism. The former effect can be mitigated with a proper geometric design, namely a phononic shield [25] or a unitary cell 
displaying a full phononic gap [23]. On the other hand, surface damping losses can be greatly reduced with proper surface treatments [26], which can be also beneficial for improving the optical Q-factor at low temperatures. In fact, it has been elucidated that key elements in determining the role of surface scattering are the atomic scale surface morphology and its chemical nature [29]. However, in view of producing a practical source, RT operation is desirable. In order to deal with the phonon-phonon scattering contribution, a challenging strategy would be to modify the lifetime of the thermal phonons by tailoring the thermal properties of the devices [28]. Thermoelastic losses could be addressed by using alternative materials, such as diamond, which is stiffer than Si. Nevertheless, the few works present in literature concerning diamond-based OM devices report values of $Q_{m, l}$ hardly above $10^{4}$ at RT [30-32].

Especially remarkable results in what concerns RT operation are those recently obtained on high-stress Si nitride membranes [58], where mechanical Q-factors of $10^{8}$ were measured under high vacuum conditions for fundamental modes above $100 \mathrm{kHz}$. Indeed, those specifications would even enable reaching ground state OM cooling from RT and hence, opening the way of performing experiments in the quantum regime without passively cooling the system

\section{Conclusions and outlook}

In this work we have reviewed several mechanisms that can drive OM cavities into a phonon lasing regime. Those can be versatile sources of coherent acoustic waves over a wide range of frequencies that promise applications in imaging, nondestructive evaluation, and medicine, as well as in other yet unexplored fields.

Conventional sources of sound waves, such as piezoelectric transducers, do not operate efficiently above few tens of gigahertz, hence chip-integrated phonon lasers able to reach those frequencies would be of great interest to the scientific community. In this sense, within the variety of $\mathrm{OM}$ cavities, we believe that $\mathrm{OM}$ crystals are the most promising platforms, given that the extraction of the eventual coherent signal is straightforward. Moreover, the continuous improvement of nanofabrication techniques reduce the minimum sizes achievable on a chip, enabling the up-scaling of the available frequencies. Indeed, state of the art Si-based phononic crystals already allows tailoring phononic bands up to the tens of gigahertz, where phonons are less sensitive to thermal decoherence. 


\section{Acknowledgements}

This work was supported by the European Comission project TAILPHOX (ICT-FP7-233883), the Spanish Severo Ochoa Excellence program and the MINECO project PHENTOM (FIS201570862-P). DNU and MFC gratefully acknowledge the support of a Ramón y Cajal postdoctoral fellowship and a Severo Ochoa studentship, respectivelyn. The authors sincerely thank B. Djafari-Rouhani, Y. Pennec, S. El-Jallal and M. Oudich for the design of the OM photonic crystal and A. Griol and A. Martinez for the fabrication of the OM photonic crystal.

\section{References}

1. Aspelmeyer M, Kippenberg T J and Marquardt F 2014 Rev. Mod. Phys. 861391

2. Vahala K J 2008 Phys. Rev. A 78(2) 023832.

3. Tallur J S, Sridaran S, Bhave S A and Carmon T 2010 Frequency Control Symposium (FCS), IEEE International 268

4. Heinrich G, Ludwig M, Qian J, Kubala B and Marquardt F 2011 Phys. Rev. Lett. 107 043603

5. Zhang M, Wiederhecker G S, Manipatruni S, Barnard A, McEuen P and Lipson M 2012 Phys. Rev. Lett. 109233906

6. Bagheri M, Poot M, Fan L, Marquardt F and Tang H X 2013 Phys. Rev. Lett. 111213902

7. Zhang M, Shah S, Cardenas J and Lipson M 2015 Phys. Rev. Lett. 115163902

8. Matheny M H et al. Phys. Rev. Lett. 2014112014101

9. Feng X L, White C J, Hajimiri A and Roukes M L 2008 Nat. Nanotech. 3342

10. Fang K, Matheny M M, Luan X and Painter O 2015 arXiv:1508.05138

11. K. J. Vahala et al., Nature Phys. 5, 682 (2009)

12. Grudinin IS, Lee H, Painter O and Vahala K J 2010 Phys. Rev. Lett. 104083901

13. Mahboob I, Nishiguchi K, Fujiwara A and Yamaguchi H 2013 Phys. Rev. Lett. 110(12) 127202

14. Schliesser A and Kippenberg T J 2014 Cavity Optomechanics, ed M Aspelmeyer, T J Kippenberg and F Marquardt (Springer: Berlin Heidelberg) c 6

15. Kippenberg T J, Rokhsari H, Carmon T, Scherer A and Vahala K J 2005 Phys. Rev. Lett. 95 033901

16. Eichenfield M 2010 Cavity optomechanics in photonic and phononic crystals: engineering the interaction of light and sound at the nanoscale Ph.D. thesis, California Institute of Technology 
17. Massel F, Heikkilä T T, Pirkkalainen J-M, Cho S U, Saloniemi H, Hakonen P J and Sillanpää M A 2011 Nature 480, 351-354

18. Arcizet O, Cohadon P F, Briant T, Pinard M and Heidmann A 2006 Nature 444 (7115) 71-74

19. Anetsberger $G$, Arcizet $O$, Unterreithmeier $Q P$, Rivière $R$, Schliesser $A$, Weig $E M$, Kotthaus J P and Kippenberg T J 2009 Nature Phys. 5 909-914

20. Kemiktarak U, Durand M, Metcalfe M and Lawall J 2014 Phys. Rev. Lett. 113030802

21. Marquardt F, Harris J G E and Girvin S M 2006 Phys. Rev. Lett. 96103901

22. Krause A G, Hill J T, Ludwig M, Safavi-Naeini A H, Chan J, Marquardt F and Painter $O$ 2015 Phys. Rev. Lett. $115233601 \mathrm{~L}$

23. Gomis-Bresco J et al. 2014 Nat. Commun. 54452

24. Navarro-Urrios D et al. 2014 AIP Advances 4124601

25. Chan J, Safavi-Naeini A H, Hill J T, Meenehan S and Painter O 2012 Appl. Phys. Lett. 101 081115

26. Chan J 2012 Laser cooling of an optomechanical crystal resonator to its quantum ground state of motion Ph.D. thesis, California Institute of Technology

27. Davanco M, Ates S, Liu Y and Srinivasan K 2014 Appl. Phys. Lett. 104(4), 041101.

28. Chávez-Ángel E, Zarate R A, Gomis-Bresco J, Alzina F and Sotomayor Torres C M 2014 Semicond. Scie. Tech. 2912124010

29. Neogi S et al. 2015 ACS Nano 943820

30. Mitchell M, Khanaliloo B, Lake D Pand Barclay P E 2015 arXiv:1511.04456

31. Burek M J et al. 2015 arXiv:1512.04166

32. Rath P, Khasminskaya S, Nebel C, Wild C and Pernice W H 2013 Nat. Commun. 4, 1690

33. Navarro-Urrios D, Capuj N E, Gomis-Bresco J, Alzina F, Pitanti A, Griol A, Martinez A and Sotomayor Torres C M 2015 Scientific Reports 515733

34. Bagheri M, Poot M, Li M, Pernice W P and Tang H X 2011 Nat. Nanotechnol. 6(11) 726732

35. Cole G D and Aspelmeyer M 2011 Nat. Nanotechnol. 6(11) 690-691.

36. Zalalutdinov M, Zehnder A, Olkhovets A, Turner S, Sekaric L, Ilic B, Czaplewski D, Parpia J M and Craighead H G 2001 Appl. Phys. Lett. 79695.

37. Höhberger C and Karrai K 2004 Proceedings of the 4th IEEE Conference on Nanotechnology (IEEE, New York).

38. Pruessner M W, Stievater T H, Khurgin J B and Rabinovich W S 2011 Opt. Express 19(22) 21904-21918 
39. Okamoto H, Ito D, Onomitsu K, Sanada H, Gotoh H, Sogawa T and Yamaguchi H 2011 Phys. Rev. Lett. 106036801

40. Bahl G, Zehnpfennig J, Tomes M and Carmon T 2011 Nat. Commun. 2403

41. Savchenkov A A, Matsko A B, Ilchenko V S, Seidel D and Maleki L 2011 Opt. Lett. 36 3338.

42. It is worth noting that here we make an adiabatic approximation for the optical response of the cavities, the complexity of the dynamics being much increased in the case of optical cavities with optical damping rates comparable with the SP limit-cycle frequencies.

43. Baker C et al. 2012 Opt. Express 20(27), 29076-29089.

44. Fomin A E, Gorodetsky M L, Grudinin I S and Ilchenko V S 2005 J. Opt. Soc. Am. B 22 459-465

45. Soltani M, Yegnanarayanan S, Li Q, Eftekhar A A and Adibi A 2012 Phys. Rev. A 85(5) 053819

46. Johnson T J, Borselli M and Painter O 2006 Opt. Express 14817

47. Xu Q and Lipson M 2006 Opt. Lett. 31341

48. Barclay P E, Srinivasan K and Painter O 2005 Opt. Express 13801

49. Pernice W H, Li M and Tang H X 2010 Opt. Express 1818438

50. Yang J, Gu T, Zheng J, Yu M, Lo G-Q, Kwong D-L and Wong C W 2014 Appl. Phys. Lett. 104061104

51. Zhang L, Fei Y, Cao Y, Lei X and Chen S 2014 JOSA B 31201

52. Deng Y, Liu F, Leseman Z and Hossein-Zadeh M 2013 Opt. Express 214653

53. Navarro-Urrios D, Tredicucci A and Sotomayor-Torres C M 2015 SPIE Newsroom DOI: $10.1117 / 2.1201507 .006036$

54. Deng et al. Opt. Express 2013214653

55. Deng Y, Flores-Flores R, Jain R K and Hossein-Zadeh M 2013 Opt. Lett. 38(21) 44134416

56. Gieseler J, Novotny L and Quidant R 2013 Nat. Phys. 9806

57. Verhagen E, Deleglise S, Weis S, Schliesser A and Kippenberg T J 2012 Nature 48263.

58. Norte R A, Moura J P, Gröblacher S 2016 Phys. Rev. Lett. 116147202 